\title{
Microencapsulação de extrato de beterraba (Beta Vulgaris L.) pelo processo de
}

\section{gelificação iônica}

\author{
Microencapsulation of beet (Beta Vulgaris L.) beet by ionic gelation proces \\ Microencapsulación de extracto de remolacga (Beta Vulgaris L.) por processo de gelificación iónica
}

Laís Priscila Cavalcante Ferreira

ORCID: https://orcid.org/0000-0002-5317-2928 Universidade Federal de Sergipe, Brasil

E-mail: laiscavalcate06@gmail.com

Anne Caroline Rocha Xavier

ORCID: https://orcid.org/0000-0001-7421-937X Universidade Federal de Sergipe, Brasil

E-mail: anne.xavier@hotmail.com

Jucenir dos Santos

ORCID: https://orcid.org/0000-0001-6911-4354 Universidade Federal de Sergipe, Brasil

E-mail: jucenirds@hotmail.com

Elma Regina Silva de Andrade Wartha

ORCID: https://orcid.org/0000-0002-7718-1490 Universidade Federal de Sergipe, Brasil E-mail: ewartha@gmail.com

Alessandra Almeida Castro Pagani

ORCID: https://orcid.org/0000-0003-4191-3888 Universidade Federal de Sergipe, Brasil E-mail: alespagani@yahoo.com.br

\begin{abstract}
Resumo
A beterraba tem atraído atenção como alimento funcional com importante efeito promotor de saúde. Sua composição tem importantes substâncias bioativas, destacando betalaínas, ácido ascórbico, carotenóides e fenólicos. A encapsulação por gelificação iônica tem se mostrado uma técnica eficaz na obtenção de produtos palatáveis e nutritivos, mascarando sabores indesejados e preservando nutrientes. Diante do exposto, o objetivo do presente estudo foi obter microcápsulas de beterraba pelo processo de gelificação iônica e verificar a estabilidade dos compostos bioativos durante o armazenamento a $\pm 5^{\circ} \mathrm{C}$ em diferentes soluções conservadoras (padrão/sem solução, solução de ácido cítrico e ácido ascórbico). A partir do extrato de beterraba realizou-se o processo de gelificação, obtendo-se as microcápsulas, armazenando-as por 28 dias em embalagens herméticas de vidro a $5 \pm 1{ }^{\circ} \mathrm{C}$ e com as soluções conservadoras. As análises realizadas foram de rendimento, peso, tamanho, cor, sólidos solúveis, umidade, cinzas, acidez, pH, vitamina C, carotenóides, fenólicos, betalaínas e antioxidante ABTS. As características físico-químicas do extrato com relação as microcápsulas, houve redução dos sólidos solúveis, da betalaína, do antioxidante, dos carotenóides e do parâmetro de cor a*. O ácido ascórbico, fenóis, cinzas, umidade e pH se mantiveram constantes. Após 21 dias de armazenamento observou-se aumento da permeabilidade da membrana de alginato, acarretando maior migração entre os compostos do meio e das microcápsulas. Conclui-se que o processo de gelificação iônica é uma tecnologia viável para a desenvolvimento de microcápsulas de beterraba, com manutenção das características nutricionais, sendo que as microcápsulas armazenadas na solução com ácido ascórbico obtiveram melhores resultados de conservação dos compostos bioativos.
\end{abstract}

Palavras-chave: Beterraba; Microencapsulação; Compostos Bioativos.

\begin{abstract}
Beet has attracted attention as a functional food with an important health-promoting effect. It presents in its composition important bioactive substances, highlighting betalains, ascorbic acid, carotenoids and phenolic acids. Ionic gelation encapsulation has been shown to be an effective technique in obtaining palatable and nutritious products masking unwanted flavors and preserving nutrients. Therefore, the objective of the present study was to obtain beet microcapsules by the process of ionic gelation and to verify the stability of bioactive compounds during refrigerated storage in different conservative solutions (standard, citric acid and ascorbic acid). From the beet extract, the ionic gelation process was carried out, obtaining the beet microcapsules, storing them for 28 days in airtight glass containers at $5 \pm 1{ }^{\circ} \mathrm{C}$ and with conservative solutions. The analyzes performed were, weight, size, color, soluble solids, moisture, ash, acidity, pH, vitamin C, carotenoids, betalains, phenolics and ABTS. The physical-chemical
\end{abstract}


characteristics of the extract in relation to beet microcapsules, there was a significant reduction in soluble solids, betalain, antioxidant capacity, carotenoids and color parameter $\mathrm{a}^{*}$. Ascorbic acid, total phenols, ash, moisture and $\mathrm{pH}$ remained constant $(5 \%)$. After 21 days of storage, an increase in the permeability of the alginate membrane was observed, causing greater migration between the compounds of the medium and the microcapsules. It is concluded that the ionic gelation process is a viable technology for the development of microcapsules of beet extract maintaining its nutritional characteristics, and the microcapsules stored in the solution with ascorbic acid had better results for bioactive compounds.

Keywords: Beet; Microencapsulation; Bioactive Compounds.

\section{Resumen}

La remolacha ha atraído la atención como un alimento funcional con un importante efecto promotor de la salud. Su composición contiene importantes sustancias bioactivas, destacando betalaínas, ácido ascórbico, carotenoides y fenólicos. Se ha demostrado que la encapsulación por gelificación iónica es una técnica eficaz para obtener productos apetitosos y nutritivos, enmascarar los sabores no deseados y preservar los nutrientes. Dado lo anterior, el objetivo de este estudio fue obtener microcápsulas de remolacha mediante el proceso de gelificación iónica y verificar la estabilidad de los compuestos bioactivos durante el almacenamiento refrigerado $\pm 5^{\circ} \mathrm{C}$ en diferentes soluciones conservadoras (estándar/sin solución, solución de ácido cítrico y de ácido ascórbico). A partir del extracto de remolacha se realizó el proceso de gelificación iónica, obteniendo las microcápsulas de remolacha, almacenándolas durante 28 días en envases de vidrio herméticos a $5 \pm 1{ }^{\circ} \mathrm{C}$ y con las soluciones conservadoras. Los análisis realizados fueron rendimiento, peso, calibre, color, sólidos solubles, humedad, ceniza, acidez, $\mathrm{pH}$, vitamina $\mathrm{C}$, carotenoides, fenólicos, betalaínas y capacidad antioxidante (ABTS). Las características fisicoquímicas del extracto en relación a las microcápsulas de remolacha, hubo reducción de sólidos solubles, betalaína, capacidad antioxidante, carotenoides y el parámetro de color $\mathrm{a}^{*}$. El ácido ascórbico, los fenoles totales, las cenizas, la humedad y el pH se mantuvieron constantes durante el almacenamiento. Luego de 21 días de almacenamiento, se observó un aumento en la permeabilidad de la membrana de alginato, provocando una mayor migración entre los compuestos del medio y las microcápsulas. Se concluye que el proceso de gelificación iónica es una tecnología viable para el desarrollo de microcápsulas de extracto de remolacha, con mantenimiento de las características nutricionales durante 28 días, y que las microcápsulas almacenadas en solución con ácido ascórbico obtuvieron mejores resultados para la conservación de compuestos bioactivos.

Palabras clave: Remolacha; Microencapsulación; Compuestos Bioactivos.

\section{Introdução}

Na última década, a beterraba (Beta vulgaris L.) tem atraído atenção como alimento funcional por ser um dos vegetais que contêm importantes compostos, como betalaínas, fibras, nitrato, ácido ascórbico, carotenóides, ácidos fenólicos e flavonóides. Diversos efeitos benéficos à saúde humana têm sido associados à essas substâncias, incluindo quimioprevenção a alguns tipos de câncer, proteção contra o estresse oxidativo e doenças correlacionadas, além de efeito vasoprotetor, redução da pressão arterial aguda e, benefícios gastrointestinais e hepatoprotetor, como também, outras propriedades funcionais incluem atividades antivirais e antimicrobianas (Dalla et al., 2015; Kluge et al., 2016 e Clifford et at., 2015).

De acordo com Clifford et al., (2015) derivados da beterraba, como o suco, preservam parte dos compostos e respectivas propriedades do vegetal e que quando se compara com sucos de outros vegetais, este se destaca com capacidade antioxidante muito superior à de sucos vegetais mais conhecidos, como tomate e cenoura, e sucos de frutas, como laranja e abacaxi, sendo apenas o suco de romã superior em capacidade antioxidante em ensaio FRAP.

Apesar da grande relevância nutricional, a beterraba ainda é pouco consumida. A presença dos compostos aromáticos, como geosmim e pirazinas, lhe confere características indesejáveis, como o de "odor terroso", o que limita o consumo do vegetal (Volp et al., 2009 e Tivelli et al., 2011). Dentro desse contexto, a técnica de encapsulação, por gelificação iônica, é eficaz para a manipulação e aumento da vida útil dos compostos de forma versátil, econômica e atóxica. A utilização de alginatos, como material encapsulante, pode resultar em um produto final que protege os compostos bioativos nutracêuticos dos alimentos encapsulados de fatores negativos, tais como umidade e calor, melhorando a estabilidade e a biodisponibilidade destes compostos e preservando os alimentos durante o processamento e armazenamento com a liberação gradual e controlada dos compostos (Pasin et al., 2012; Morais et al., 2015; Morais et al., 2017). 
A encapsulação também facilita o manuseio de produtos líquidos ou gasosos que, a partir dessa técnica, permanecem na forma sólida. Além, da possibilita de mascarar gosto de componentes desagradáveis, o que suaviza sabores indesejados (Fávaro-Trindade et al., 2008; Fang Z et al., 2010; Mirzaei et al., 2012). A utilização de alginatos destaca-se não apenas pelo seu uso técnico e econômico, mas como fator de saúde, promovendo a redução dos níveis de açúcar e colesterol no sangue devido a sua classificação nutricional como fibra insolúvel (Pasin et al., 2012). Dentro desse contexto, a microencapsulação por gelificação iônica com alginato destaca-se como uma técnica promissora para preservar os compostos de interesse nutricionais da beterraba e, aumentar seu consumo por meio de um novo tipo de produto. Portanto, o objetivo do presente estudo foi obter microcápsulas de extrato de beterraba pelo processo de gelificação iônica e, verificar a estabilidade fisicoquímica, fitoquímica e capacidade antioxidante durante o armazenamento refrigerado $\left(5 \pm 1^{\circ} \mathrm{C}\right)$ por 28 dias em diferentes soluções.

\section{Metodologia}

As beterrabas, originárias de Irecê-BA e colhidas com 90 dias, foram adquiridas na Central de Abastecimento do Estado de Sergipe (CEASA), na cidade de Aracaju/BRASIL. As matérias-primas apresentaram coloração uniforme, cascas íntegras e tamanho médio de 255g. A higienização foi realizada de acordo com Silva et al., (2016) e, em seguida, as beterrabas foram fracionadas e processadas em centrífuga juicer Mondial ${ }^{\circledR}$ 300W para obtenção do extrato concentrado.

As microcápsulas do extrato de beterraba foram obtidas por meio da técnica de gelificação iônica com alginato de sódio e cloreto de cálcio, utilizando o método de gotejamento e concentrações de reagentes de acordo com Pagani et al., (2014) (Figura 1). Depois de formadas, as microcápsulas ficaram em repouso na solução 2 por aproximadamente 1 minuto, tempo observado previamente para solidificação das microcápsulas, e com o auxílio de peneira, foram drenadas e imersas em recipiente com água destilada para a retirada de possíveis resíduos da solução 2 (Figura 2).

Figura 1: Esquema da gelificação iônica por gotejamento

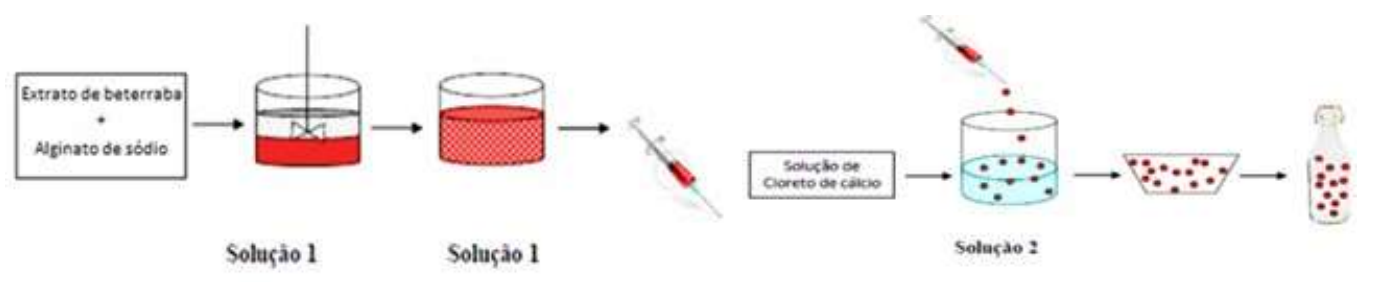

Fonte: Pagani et al. (2014).

Figura 2: Microcápsulas de extrato de beterraba obtidas pelo processo de gelificação iônica

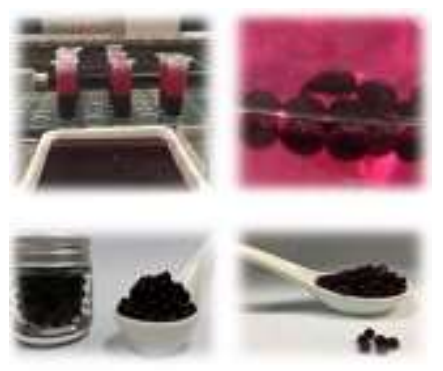

Fonte: Autores (2021). 
Após a lavagem e drenagem, amostras de 50g de microcápsulas foram armazenadas em três diferentes tratamentos:

- $\quad$ Controle: Padrão, apenas as microcápsulas;

- Tratamento 1: 20ml de Ácido ascórbico (0,04\% - pH 4,57)

- Tratamento 2: $20 \mathrm{ml}$ de Ácido cítrico (0,15\% - pH 3,84).

$\mathrm{O}$ armazenamento ocorreu em recipiente de vidro com tampa hermética com capacidade de $50 \mathrm{ml}$ à temperatura de refrigeração $\left(5 \pm 1^{\circ} \mathrm{C}\right)$ por 28 dias. As amostras foram analisadas em intervalos de 7 dias em 5 pontos $(0,7,14,21$ e 28 dias).

\subsection{Análises Físico-Químicas}

Foram efetuadas análises para caracterização físico-química do extrato e das microcápsulas de beterraba. O rendimento foi obtido pesando o extrato da beterraba antes do processamento e as microcápsulas após serem processadas, calculando-se a razão entre a diferença multiplicada por 100 de acordo com Oliveira (2011). O tamanho das microcápsulas de beterraba foi avaliado utilizando um micrômetro digital Pantec®. Para mensuração do peso, foi utilizada a balança digital de capacidade de $0,001 \mathrm{~g}-30 \mathrm{~g}$. O teor de umidade foi determinado pelo método de secagem direta em estufa a $105{ }^{\circ} \mathrm{C}$ até peso constante, seguindo o método 012/IV do Instituto Adolfo Lutz (2005). Foi utilizado o método de incineração em mufla para a análise de cinzas segundo metodologia descrita pela AOAC (1996). Para determinação do pH foi utilizado o método Potenciométrico seguindo metodologia 017/IV do Instituto Adolfo Lutz (2005). O teor de acidez foi determinado pelo método de acidez titulável determinada de acordo com AOAC (1996) e expressa em porcentagem de ácido cítrico. Para a determinação de sólidos solúveis foi avaliada em refratômetro Abbé de bancada (Biobrix) e o resultado expresso em ${ }^{\circ}$ Brix. As leituras da cor foram obtidas com a utilização do colorímetro Color Meter Minolta 200b de acordo com Palou et al. (1999). Os teores de ácido ascórbico foram avaliados seguindo o método proposto por Oliveira et al., (2010) e expressos em mg/g.

\subsection{Análise dos Compostos Bioativos Nutracêuticos}

Para quantificação das betalaínas, foi utilizada a metodologia de Giusti e Wrolstad (2001) adaptada. A leitura foi realizada na faixa de $520 \mathrm{~nm}$ (referente à betanina) e os resultados expressos em mg/100g.

Os teores de carotenóides totais foram avaliados seguindo o método proposto por Lichtenthaler (1987), os resultados foram expressos em $\mu \mathrm{g} / \mathrm{g}$.

Os compostos fenólicos foram obtidos mediante extração alcoólica e absorbância de $725 \mathrm{~nm}$ em espectrofotômetro de acordo com Kim et al. (2003), em mg GAE/g.

\subsection{Capacidade Antioxidante}

A análise antioxidante pelo ensaio de ABTS foi realizada baseada na metodologia de Rufino et al. (2007) com adaptações, realizando a determinação direta através da inserção dos resultados diretamente na curva do ABTS em $\mu$ mol TEAC/g.

\subsection{Delineamento Experimental e Análise Estatística}

Os experimentos foram realizados segundo o delineamento inteiramente casualizado (DIC). Para detecção das diferenças entre tratamentos e o controle em função do tempo foi utilizado o delineamento fatorial cruzado. Foram realizadas três repetições em triplicata e os dados expressos como médias para melhor precisão dos resultados das análises. Os dados foram submetidos à análise de variância (ANOVA) e teste de Tukey a 5\% de probabilidade, utilizando o software ASSISTAT 
(Assistência Estatística), versão 7.7, beta, para detecção de diferenças de médias entre o extrato e as microcápsulas processadas.

\section{Resultados e Discussão}

O rendimento em microcápsula de beterraba foi de $65 \%$ em relação ao extrato. Em estudos semelhantes com gelificação iônica de polpa de fruta observou-se valores próximos, embora ligeiramente superiores. O estudo de Xavier (2014) com gelificação iônica de polpa de maracujá obteve 70\% de rendimento e o estudo de Oliveira (2011) com a gelificação iônica da polpa de mamão obteve $75,95 \%$. Essa leve diferença deve-se, possivelmente, a densidade do extrato, o qual dificultou a formação das microcápsulas.

O presente estudo quantificou o peso e tamanho médio de $0,0012 \mathrm{~g}$ e $3,88 \mathrm{~mm}$, respectivamente, caracterizando-se assim como microcápsulas, segundo o estudo de Baker (1986) que classifica as cápsulas pelo tamanho em 3 categorias: macro( $>5000 \mu \mathrm{m})$, micro- $(0,2-5000 \mu \mathrm{m})$ e nanocápsulas $(<0,2 \mu \mathrm{m})$.

A Tabela 1 apresenta os resultados das análises físico-químicas do extrato e das microcápsulas de beterraba para comparação das mudanças ocorridas após o processamento.

Observando os resultados obtidos na Tabela 1, é possível verificar que para a maioria dos parâmetros não foram observadas diferenças significativas entre o extrato e as microcápsulas.

Tabela 1: Caracterização físico-química do extrato e das microcápsulas de beterraba recém-processadas.

\begin{tabular}{lcc}
\hline \multicolumn{1}{c}{ Análises } & Extrato de beterraba & Microcápsulas de beterraba \\
\hline Umidade $(\%)$ & $93,013 \pm 0,130^{\mathrm{a}}$ & $92,697 \pm 0,270^{\mathrm{a}}$ \\
Cinzas $(\%)$ & $5,840 \pm 1,820^{\mathrm{a}}$ & $5,939 \pm 2,120^{\mathrm{a}}$ \\
$\mathrm{pH}$ & $5,920 \pm 0,010^{\mathrm{a}}$ & $5,970 \pm 0,050^{\mathrm{a}}$ \\
Sólidos Solúveis $\left({ }^{\circ}\right.$ Brix) & $7,33 \pm 0,070^{\mathrm{a}}$ & $4 \pm 0,000^{\mathrm{b}}$ \\
Acidez (g ácido cítrico/100g) & $0,053 \pm 0,008^{\mathrm{b}}$ & $0,083 \pm 0,001^{\mathrm{a}}$ \\
Ácido ascórbico $(\mathrm{mg} / 100 \mathrm{~g})$ & $63,295 \pm 2,090^{\mathrm{a}}$ & $63,567 \pm 1,26^{\mathrm{a}}$ \\
Carotenóides $(\mu \mathrm{g} / \mathrm{g})$ & $155,614 \pm 6,307^{\mathrm{a}}$ & $31,028 \pm 4,37^{\mathrm{b}}$ \\
Fenóis $(\mathrm{mg}$ GAE/g) & $82,391 \pm 17,356^{\mathrm{a}}$ & $64,952 \pm 6,14^{\mathrm{a}}$ \\
Betalaína $(\mathrm{mg} / 100 \mathrm{~g})$ & $176,04 \pm 1,21^{\mathrm{a}}$ & $131,34 \pm 11,77^{\mathrm{b}}$ \\
Cor $\quad \mathrm{L}^{*}$ & $26,633 \pm 3,730^{\mathrm{a}}$ & $26,80 \pm 3,25^{\mathrm{a}}$ \\
$\quad \mathrm{a}^{*}$ & $2,633 \pm 0,260^{\mathrm{a}}$ & $1,60 \pm 0,26^{\mathrm{b}}$ \\
$\quad \mathrm{b}^{*}$ & $10,567 \pm 0,210^{\mathrm{a}}$ & $10,567 \pm 0,52^{\mathrm{a}}$ \\
$\quad \mathrm{c}^{*}$ & $10,867 \pm 0,550^{\mathrm{a}}$ & $10,530 \pm 0,21^{\mathrm{a}}$ \\
$\quad \mathrm{H}$ & $76,033 \pm 2,570^{\mathrm{b}}$ & $81,267 \pm 1,86^{\mathrm{a}}$ \\
ABTS $(\mu \mathrm{mol} \mathrm{TEAC/g)}$ & $205,08 \pm 13,760^{\mathrm{a}}$ & $138,287 \pm 13,46^{\mathrm{b}}$ \\
\hline
\end{tabular}

Médias na mesma linha seguidas pela mesma letra não diferem estatisticamente entre si a 5\% de probabilidade. Fonte: Autores (2021)

O teor de umidade do extrato (líquido) e das microcápsulas (sólido) foram muito próximos, apesar da diferença física entre ambos. Morais et al., (2017) em seu estudo de gelificação com polpa de murici também observou dados semelhantes e atribuiu tal resultado a deficiência do processo de drenagem, uma vez que durante as etapas do processamento as microcápsulas são imersas em água para retirada do excesso de cloreto.

Em relação ao teor de cinzas, também não foram observados resultados estatisticamente diferentes entre extrato e microcápsulas de beterraba. Morais et al., (2015) e Oliveira (2011) observaram, em seus estudos de gelificação iônica, 
resultado levemente superior para as microcápsulas, o que também foi encontrado no presente estudo. Os autores atribuem tal fato à incorporação do alginato e cálcio, necessários no processo de microencapsulação.

Os dados médios de $\mathrm{pH}$ foram de 5,92 para o extrato e 5,97 para a microcápsulas, sendo estes bastante semelhantes a $5 \%$ de probabilidade. A constância do pH é de extrema importância para estabilidade da betalaína, visto que a manutenção de suas características depende da faixa de $\mathrm{pH}$ (excelente estabilidade entre $\mathrm{pH} 4$ e 5 na ausência de oxigênio, e entre pH 5 e 6 e presença de oxigênio) Volpe et al., (2009) e Schiozer et al., (2013).

Em relação ao ${ }^{\circ}$ Brix, as microcápsulas apresentaram valores inferiores em relação ao extrato $(p \geq 0,05)$, resultados semelhantes foram encontrados nos estudos de Morais et al., (2015), Silva et al., (2012) e Oliveira (2011), com microencapsulação por gelificação iônica de maracujá, melancia e mamão, respectivamente. O que pode ser justificado pela possível perda de sólidos solúveis durante a imersão na solução de cloreto de cálcio e depois em água para remoção do excesso de cloreto.

Quanto ao teor de acidez, tanto para o extrato quanto para as microcápsulas de beterraba, foram observados valores próximos aos encontrados por Marques et al., (2010), 0,09. Neto et al., (2017) e Lacerda (2014) observou valores de acidez entre $0,063-0,69$ e $0,20-0,23$, respectivamente.

Comparando-se os teores de ácido ascórbico do extrato $(63,30 \mathrm{mg} / 100 \mathrm{~g})$ com as microcápsulas $(63,57 \mathrm{mg} / 100 \mathrm{~g})$, observa-se que não ocorreu diferença significativa $(\mathrm{p} \leq 0,05)$ entre as amostras. Resultados semelhantes foram observados nos estudos de Morais et al., (2014), com gelificação de cambuí, onde o teor de ácido ascórbico permaneceu constante. Tal resultado é extremamente benéfico, pois além de um importante antioxidante, sua alta instabilidade torna essa vitamina um marcador de qualidade sensorial e nutricional Bosch et al., (2013).

O teor de carotenóides totais do extrato foi de $155,61 \mu \mathrm{g} / 100 \mathrm{~g}$, enquanto que o da microcápsula foi de $31,03 \mu \mathrm{g} / 100^{-1} \mathrm{~g}$, significativamente inferior, demonstrando que tal compostos podem ter sido perdidos durante o processamento ou que a membrana de alginato tenha dificultado sua extração, agindo como interferente nas análises desses compostos. Silva et al. (2012), em seu estudo com a gelificação de polpa de melancia observou que o teor de carotenóides do produto gelificado foi inferior ao da polpa. O estudo de Oliveira (2011) e Morais et al., (2014), com mamão e cambuí, respectivamente, também apresentaram resultados diferentes para polpa e as microcápsulas, sendo a polpa de valor superior. Nos estudos de Passos (2017) e Morais et al., (2015), com microcápsulas de tomate e de maracujá, os valores de carotenóides para polpa e microcápsulas não diferiram entre si. O diferente comportamento dos carotenóides é justificado pelas metodologias utilizadas e, principalmente pela diferença dos alimentos. O aprisionamento dos compostos bioativos e, consequentemente, a sua manutenção será diferente devido a matriz alimentar única de cada espécie.

Em relação ao teor de compostos fenólicos, não foi observada diferença significativa $(p>0,05)$ entre extrato $(82,34 \mathrm{mg})$ e microcápsulas (64,95mg), indicando que este composto não é alterado pelo o processo de gelificação iônica. Passos (2017), em seu estudo com gelificação de polpa de tomate, também não observou alteração nos níveis de fenólicos após o processamento das microcápsulas de polpa tomate pela técnica de gelificacão iônica. Nos estudos de Morais et al., (2015) e Xavier (2014), no entanto, a gelificação iônica influenciou significativamente na redução dos compostos fenólicos. Possivelmente a adição de água à polpa de maracujá para o processamento das microcápsulas tenha influenciado nos teores de fenólicos.

Em relação a cor, quando comparados os parâmetros da beterraba in natura $\left(a^{*}=+2,63 ; b^{*}=+10,57 ; \mathrm{L}^{*}=26,63\right)$ com os parâmetros da microcápsula $\left(a^{*}=+1,60 ; b^{*}=+10,57 ; \mathrm{L}^{*}=26,80\right)$ podemos observar que após o processo de gelificação iônica as microcápsulas apresentaram menor valor de $a^{*}$, tal fato pode ser explicado pela redução da betalaína (176,04 $131,34 \mathrm{mg} / 100 \mathrm{~g}^{-1}$ ) durante o processamento. Além de muito instável na presença de luz e oxigênio, a betalaína pode ser facilmente lixiviada em água, pois é altamente hidrossolúvel. Os processos de sanitização favorecem a perda desses pigmentos, 
sendo facilmente perdidos quando em contato com meios aquosos Vitti (2003). Assim dito, durante duas etapas do processamento, as microcápsulas foram drenadas, justificando possivelmente tal perda Volp et al., (2009).

O parâmetro de cor H aumentou após a encapsulação, indicando aumento da coloração amarela. Tal fato indica, possivelmente, que durante o processamento houve degradação das betalaínas, liberando assim ácido betalâmico, caracteristicamente amarelo, e aumentando o ângulo Hue para valores mais próximos a 90 Volp et al., (2009). Outra possível explicação seria pela presença das betaxantinas, fração das betalaínas responsáveis pela pigmentação amarela Dalla (2015). Com a degradação das becianinas, as betaxantinas, possivelmente, foram evidenciadas nas análises de cor.

Quanto a análise antioxidante pelo método ABTS, quando comparado o extrato $(205,080 \mu \mathrm{mol})$ com as microcápsulas $(138,287 \mu \mathrm{mol}$ TEAC/g), observou-se redução significativa do parâmetro. Sendo os compostos bioativos avaliados os principais responsáveis pela atividade antioxidante da beterraba, tal resultado é condizente com os dados observados, onde apesar da estabilidade da vitamina $\mathrm{C}$ e dos compostos fenólicos, houve redução significativa das betalaínas e dos carotenóides, acarretando assim na redução da atividade antioxidante Clifford et al., (2015), Holkem et al., (2015) e Singh et al., (2017).

Na Tabela 2 estão expostos os resultados das análises físico-químicas durante o armazenamento por 28 dias a $5 \pm 1^{\circ} \mathrm{C}$ em diferentes meios ácidos e no controle (Padrão).

Tabela 2: Análises físico-químicas das microcápsulas de beterraba armazenadas em diferentes meios a $5 \pm 1^{\circ} \mathrm{C}$ durante 28 dias em embalagens de vidro.

\begin{tabular}{|c|c|c|c|c|c|c|}
\hline \multirow[t]{2}{*}{ Análise } & \multirow{2}{*}{$\begin{array}{c}\text { Meio de } \\
\text { armazenamento }\end{array}$} & \multicolumn{5}{|c|}{ Tempo (dias) } \\
\hline & & $\mathbf{0}$ & 7 & 14 & 21 & 28 \\
\hline \multirow{3}{*}{ 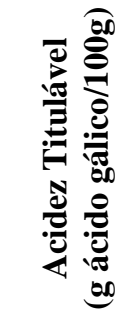 } & Padrão & $0,07 \pm 0,03 \mathrm{aA}$ & $0,047 \pm 0,01 \mathrm{abAB}$ & $0,02 \pm 0,000 \mathrm{bA}$ & $0,03 \pm 0,01 \mathrm{abB}$ & $0,02 \pm 0,007 \mathrm{bB}$ \\
\hline & Ácido ascórbico & $0,07 \pm 0,03 \mathrm{aA}$ & $0,028 \pm 0,009 \mathrm{bB}$ & $0,03 \pm 0,005 \mathrm{bA}$ & $0,074 \pm 0,03 \mathrm{Aa}$ & $0,05 \pm 0,07 \mathrm{abA}$ \\
\hline & Ácido cítrico & $0,07 \pm 0,03 \mathrm{aA}$ & $0,066 \pm 0,03 \mathrm{aA}$ & $0,04 \pm 0,026 \mathrm{aA}$ & $0,06 \pm 0,01 \mathrm{aAB}$ & $0,07 \pm 0,01 \mathrm{aA}$ \\
\hline \multirow{3}{*}{ 졸 } & Padrão & $5,97 \pm 0,05 \mathrm{aA}$ & $5,50 \pm 0,03 \mathrm{bA}$ & $5,16 \pm 0,29 \mathrm{bA}$ & $4,95 \pm 0,17 \mathrm{bA}$ & $5,12 \pm 0,21 \mathrm{bA}$ \\
\hline & Ácido ascórbico & $5,97 \pm 0,05 \mathrm{aA}$ & $5,85 \pm 0,02 \mathrm{aA}$ & $4,57 \pm 0,14 \mathrm{bB}$ & $4,58 \pm 0,15 \mathrm{aA}$ & $4,05 \pm 0,08 \mathrm{cB}$ \\
\hline & Ácido cítrico & $5,97 \pm 0,05 \mathrm{aA}$ & $4,53 \pm 0,19 \mathrm{bB}$ & $4,07 \pm 0,43 \mathrm{cC}$ & $4,57 \pm 0,09 \mathrm{bA}$ & $4,38 \pm 0,17 \mathrm{bcB}$ \\
\hline \multirow{3}{*}{ 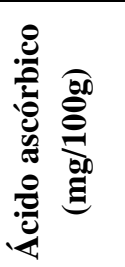 } & Padrão & $63,57 \pm 1,21 \mathrm{aA}$ & $48,35 \pm 0,95 \mathrm{bA}$ & $32,67 \pm 0,39 \mathrm{bB}$ & $42,43 \pm 0,12 \mathrm{bAB}$ & $63,64 \pm 0,29 \mathrm{Aa}$ \\
\hline & Ácido ascórbico & $63,57 \pm 1,21 \mathrm{aA}$ & $64,13 \pm 0,55 \mathrm{Aa}$ & $65,24 \pm 0,27 \mathrm{aA}$ & $65,43 \pm 1,21 \mathrm{aA}$ & $96,01 \pm 3,21 \mathrm{Bb}$ \\
\hline & Ácido cítrico & $63,57 \pm 1,21 \mathrm{aA}$ & $31,06 \pm 0,24 \mathrm{bAB}$ & $49,53 \pm 0,23 \mathrm{bAB}$ & $32,66 \pm 0,43 \mathrm{bB}$ & $65,33 \pm 0,03 \mathrm{Aa}$ \\
\hline
\end{tabular}

Médias seguidas pela mesma letra, minúscula na linha e maiúscula na coluna, não diferem entre si pelo teste de Tukey ao nível de 5\% de probabilidade.

Fonte: Autores, (2021)

Para o teor de acidez titulável (ATT), houve redução ao decorrer do tempo, com diferença significativa para o tratamento Padrão (Tabela 2). Passos (2017), Xavier et al., (2016) e Oliveira (2011) também observaram em seus estudos com gelificação iônica de polpa de tomate, de maracujá e de mamão, respectivamente, redução da acidez ao longo do armazenamento. 
Os valores de $\mathrm{pH}$ durante o período de armazenamento de 28 dias (Tabela 3) apresentaram redução significativa nos tratamentos e no padrão. Entretanto, segundo Schiozer et al., (2013) os valores ainda permaneceram dentro da faixa ideal (4 6) de estabilidade do pigmento betalaína.

Quanto aos teores de Vitamina C, constatou-se que houve oscilação durante o armazenamento para o Padrão e o tratamento com Ácido Cítrico (Tabela 4). Para o tratamento com Ácido Ascórbico, os valores permaneceram estáveis até o $21^{\circ}$ dia. Segundo Sezer et al., (1999) e Aranha (2015), microcápsulas de alginato formadas por gelificação iônica apresentam elevada porosidade, consequentemente alto índice de difusão, podendo acelerar a liberação do material microencapsulado.

No $28^{\circ}$ dia observou-se aumento dos teores de ácido ascórbico para os tratamentos e o Padrão. Tais resultados sugerem aumento da porosidade da membrana, acarretando maior interação entre o meio e as microcápsulas, devido ao processo de difusão passiva. Aranha (2015) e Krasaekoopt et al., (2003) em seus estudos relatam que valores muito baixos de $\mathrm{pH}$ podem afetar a integridade do gel de alginato, tornando-o suscetível à desintegração. Possivelmente a redução do pH no $28^{\circ}$ dia (Tabela 3) tenha levado a perda da eficiência da membrana, aumentando a porosidade da mesma. $\mathrm{O}$ tratamento utilizando o ácido ascórbico obteve resultados estáveis durante o armazenamento nos primeiros 21 dias e aumento significativamente superior no $28^{\circ}$ (Tabela 4), indicando que a utilização do ácido ascórbico como meio de conservação foi uma estratégia eficaz na manutenção da concentração de ácido ascórbico do produto. Funcionando ainda como meio de bioativação das microcápsulas após aumento da porosidade da membrana de alginato. Segundo Morais et al., (2015), a velocidade de migração e/ou interação de compostos entre microcápsulas e o meio depende da composição do alimento estudado. Tal interação constitui a bioativação de produtos onde os compostos são encontrados em pouca ou nenhuma quantidade e pode ser realizada adicionando alimentos ricos em compostos bioativos em outros alimentos, onde estes compostos não são encontrados Morais et al., (2015). De acordo com Sucupira et al., (2015) a perda do ácido ascórbico durante o armazenamento é comumente observada em produtos, devido a facilidade de degradação pelo calor, oxidação, armazenamento, aplicação do frio e alcalinidade do meio. Morais et al., (2017), Xavier et al., (2016) e Oliveira (2011) observaram, em seus estudos com gelificação iônica, significante redução da ácido ascórbico durante o armazenamento refrigerado. A utilização de ácido ascórbico como método de conservação foi uma estratégia favorável para manutenção dos teores de vitamina C do encapsulado durante o armazenamento e como bioativação das microcápsulas, elevando os teores de vitamina ao final do armazenamento. Tal resultado é de fundamental importância, pois sabe-se que a preservação desse composto está fortemente relacionada a manutenção da capacidade antioxidante de acordo com Sucupira et al., (2015) e Bendich et al., (1995).

Em relação a colorimetria (Tabela 3), observou-se durante o período de armazenamento variação do parâmetro de $\boldsymbol{a}^{*}$ entre as amostras, independente do tratamento, indicando redução do pigmento nas microcápsulas. O parâmetro $\boldsymbol{b}^{*}$ também apresentou variação entre as amostras independente do tratamento, observando aumento para o tratamento com Ácido ascórbico ao fim do armazenamento. De acordo com Volp et al., (2009) e Volp et al., (2013) o aumento desse parâmetro pode ser decorrente das betaxantinas, responsável a coloração amarela e que podem ter sobressaído com a diminuição das betacianinas. 
Tabela 3: Parâmetros de Cor das microcápsulas de beterraba armazenadas em diferentes meios a $5 \pm 1^{\circ} \mathrm{C}$ durante 28 dias em embalagens de vidro.

\begin{tabular}{lllllll}
\hline Meio de & Cor & \multicolumn{5}{c}{ TEMPO (DIAS) } \\
\cline { 3 - 7 } armazenamento & & \multicolumn{1}{c}{$\mathbf{0}$} & \multicolumn{1}{c}{$\mathbf{7}$} & $\mathbf{1 4}$ & $\mathbf{2 1}$ & $\mathbf{2 8}$ \\
\hline Padrão & $\mathrm{L}^{*}$ & $24,80 \pm 3,27$ & $26,63 \pm 2,27$ & $27,17 \pm 2,27$ & $25,60 \pm 2,12$ & $25,80 \pm 3,23$ \\
& $\mathrm{a}^{*}$ & $1.60 \pm 0,22$ & $1.60 \pm 0,26$ & $0.87 \pm 0,14$ & $1.63 \pm 0,24$ & $1.33 \pm 0,36$ \\
& $\mathrm{~b}^{*}$ & $10.40 \pm 0,52$ & $11.20 \pm 0,38$ & $9.53 \pm 0,48$ & $10.67 \pm 0,49$ & $11.37 \pm 0,65$ \\
& $\mathrm{c}^{*}$ & $10.53 \pm 0,52$ & $12.83 \pm 0,40$ & $9.60 \pm 0,85$ & $10.80 \pm 0,82$ & $11.47 \pm 0,42$ \\
& $\mathrm{H}$ & $81.27 \pm 1,57$ & $77.40 \pm 1,57$ & $84.63 \pm 1,57$ & $81.20 \pm 2,97$ & $82.67 \pm 1,57$ \\
\hline Ácido ascórbico & $\mathrm{L}^{*}$ & $24.80 \pm 3,40$ & $26.50 \pm 2,12$ & $26.90 \pm 3,15$ & $24.97 \pm 3,27$ & $23.53 \pm 2,15$ \\
& $\mathrm{a}^{*}$ & $1.60 \pm 0,26$ & $1.53 \pm 0,21$ & $1.13 \pm 0,26$ & $1.60 \pm 0,22$ & $1.27 \pm 0,46$ \\
& $\mathrm{~b}^{*}$ & $10.40 \pm 0,47$ & $11.10 \pm 0,50$ & $9.20 \pm 0,52$ & $13.10 \pm 0,52$ & $15.63 \pm 0,52$ \\
& $\mathrm{C}^{*}$ & $10.53 \pm 0,92$ & $11.23 \pm 0,64$ & $9.27 \pm 0,54$ & $13.43 \pm 0,52$ & $15.70 \pm 0,39$ \\
& $\mathrm{H}$ & $81.27 \pm 2,57$ & $82.13 \pm 1,80$ & $81.97 \pm 1,87$ & $77.37 \pm 1,57$ & $84.83 \pm 2,65$ \\
\hline Ácido cítrico & $\mathrm{L}^{*}$ & $24.80 \pm 3,27$ & $25.07 \pm 3,27$ & $26.90 \pm 3,15$ & $26.47 \pm 2,15$ & $26.40 \pm 1,20$ \\
& $\mathrm{a}^{*}$ & $1.60 \pm 0,22$ & $1.07 \pm 0,22$ & $1.60 \pm 0,15$ & $0.97 \pm 0,24$ & $1.40 \pm 0,22$ \\
& $\mathrm{~b}^{*}$ & $10.40 \pm 0,48$ & $11.13 \pm 0,52$ & $10.77 \pm 0,70$ & $9.87 \pm 0,48$ & $10.20 \pm 0,65$ \\
& $\mathrm{C}^{*}$ & $10.53 \pm 0,48$ & $1.17 \pm 0,52$ & $11.20 \pm 0,60$ & $9.90 \pm 0,75$ & $10.50 \pm 0,75$ \\
& $\mathrm{H}$ & $81.27 \pm 1,57$ & $84.37 \pm 1,57$ & $76.10 \pm 1,57$ & $85.03 \pm 1,87$ & $80.40 \pm 2,84$ \\
\hline
\end{tabular}

Médias \pm desvio padrão

Fonte: Autores (2021).

A Tabela 4 apresenta os resultados para os compostos bioativos nutracêuticos analisados. Com relação aos teores de carotenóides totais, nesta pesquisa, foi possível verificar diminuição com o decorrer do armazenamento até o $21^{\circ}$ dia, sendo: 92,25\% para o Padrão; 94,13\% para o tratamento Ácido ascórbico e 91,56\% para o Ácido cítrico. Xavier et al., (2016), em seu estudo com microcápsulas de maracujá, observou redução de 78,79\% do teor de carotenóides no $14^{\circ}$ dia de armazenamento refrigerado. Já Oliveira (2011) detectou diminuição significativa no teor de carotenóides totais (9,94\%), nas amostras de gotas de mamão obtidas por gelificação iônica, a partir do $14^{\circ}$ de armazenamento a $5^{\circ} \mathrm{C}$. Morais et al., (2014), com microencapsulação de polpa de cambuí, também observou redução dos carotenóides totais. Entretanto, Passos (2017), em seu estudo com microcápsulas de tomate armazenadas no azeite, não observou redução. Segundo o autor, o meio de armazenamento, rico em carotenóides, pode ter favorecido a preservação desses compostos. Quanto aos carotenóides avaliados neste estudo, verificou-se resultados semelhante aos observados para o ácido ascórbico, com oscilação ao longo do armazenamento e aumento no $28^{\circ}$ dia. Possivelmente o aumento da porosidade da membrana de alginato, acarretada pela a redução do $\mathrm{pH}$, tenha ocasionado maior difusão dos compostos das microcápsulas e do meio, permitindo maior interação/migração desses compostos Aranha (2015), Krasaekoopt et al., (2003) e Sezer et al., (1999).

Quanto ao teor de fenólicos, observou-se redução em função do tempo, com influência significativa do meio de armazenamento (Tabela 4). O tratamento com Ácido ascórbico e o Padrão obtiveram, respectivamente, redução de 61,51\% e 66,79\% em relação ao teor inicial. Segundo Raupp et al., (2011), a adição de ácido cítrico preserva os compostos fenólicos e a atividade antioxidante em beterraba. Entretanto, o presente estudo não observou eficácia na conservação dos fenólicos no tratamento com Ácido cítrico, pois este apresentou redução durante o armazenamento de 61,2\%. 
Tabela 4: Concentração dos compostos bioativos nutracêuticos das microcápsulas de beterraba armazenadas em diferentes meios a $5 \pm 1^{\circ} \mathrm{C}$ durante 28 dias em embalagens de vidro.

\begin{tabular}{|c|c|c|c|c|c|c|}
\hline \multirow[t]{2}{*}{ Análise } & \multirow{2}{*}{$\begin{array}{l}\text { Meio de } \\
\text { armazenamento }\end{array}$} & \multicolumn{5}{|c|}{ Tempo (Dias) } \\
\hline & & ito & 7 & 14 & 21 & 28 \\
\hline \multirow{3}{*}{ 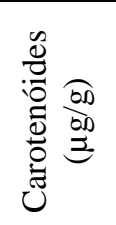 } & Padrão & $31,03 \pm 4,37 \mathrm{aA}$ & $3,32 \pm 0,50 \mathrm{bcA}$ & $7,23 \pm 1,95 \mathrm{bcA}$ & $2,40 \pm 0,57 \mathrm{cA}$ & $10,79 \pm 0,73 \mathrm{bA}$ \\
\hline & Ác. ascórbico & $31,03 \pm 4,37 \mathrm{aA}$ & $3,76 \pm 0,20 \mathrm{cA}$ & $4,59 \pm 1,28 \mathrm{cA}$ & $1,82 \pm 0,54 \mathrm{cA}$ & $11,45 \pm 1,67 \mathrm{bA}$ \\
\hline & Ác. cítrico & $31,03 \pm 4,37 \mathrm{aA}$ & $3,68 \pm 0,75 \mathrm{cA}$ & $2,84 \pm 0,60 \mathrm{cA}$ & $2,62 \pm 0,18 \mathrm{cA}$ & $10,13 \pm 0,96 \mathrm{bA}$ \\
\hline \multirow{3}{*}{ 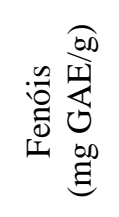 } & Padrão & $64,95 \pm 1,30 \mathrm{aA}$ & $16,67 \pm 1,30 \mathrm{cA}$ & $18,61 \pm 4,62 \mathrm{bcA}$ & $27,16 \pm 4,35 \mathrm{bA}$ & $21,57 \pm 3,34 \mathrm{bcA}$ \\
\hline & Ác. ascórbico & $64,95 \pm 1,30 \mathrm{aA}$ & $13,16 \pm 2,82 \mathrm{cA}$ & $19,48 \pm 1,61 b c A$ & $23,33 \pm 2,05 \mathrm{bA}$ & $25,00 \pm 1,92 \mathrm{bA}$ \\
\hline & Ác. cítrico & $64,95 \pm 1,30 \mathrm{aA}$ & $9,99 \pm 2,97 \mathrm{cdA}$ & $13,78 \pm 1,09 \mathrm{bcA}$ & $21,72 \mathrm{bA} \pm 3,49$ & $25,2 \pm 0,82 \mathrm{~dB}$ \\
\hline \multirow{3}{*}{ 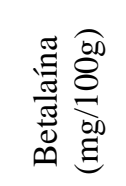 } & Padrão & $135,24 \pm 1,21 \mathrm{aA}$ & $66,51 \pm 6,11 \mathrm{bA}$ & $64,37 \pm 0,53 \mathrm{bA}$ & $39,13 \pm 7,22 \mathrm{cA}$ & $38,61 \pm 7,55 \mathrm{cA}$ \\
\hline & Á. ascórbico & $135,24 \pm 1,21 \mathrm{aA}$ & $74,78 \pm 5,94 \mathrm{bA}$ & $62,78 \pm 1,16 \mathrm{bA}$ & $50,21 \pm 6,78 \mathrm{cA}$ & $44,75 \pm 1,64 \mathrm{cA}$ \\
\hline & Ác. cítrico & $135,24 \pm 1,21 \mathrm{aA}$ & $67,56 \pm 7,71 \mathrm{bA}$ & $53,80 \pm 5,27 \mathrm{bA}$ & $51,21 \pm 2,90 \mathrm{cA}$ & $45,96 \pm 6,99 \mathrm{cA}$ \\
\hline
\end{tabular}

Médias seguidas pela mesma letra, minúscula na linha e maiúscula na coluna, não diferem entre si pelo teste de Tukey ao nível de 5\% de probabilidade

Fonte: Autores (2021)

Na segunda semana de armazenamento, foi possível observar redução dos compostos fenólicos nos tratamentos e no Padrão, com posterior aumento nas semanas seguintes. Xavier (2016), com microcápsulas de maracujá e Morais et al., (2014), com microcápsulas de cambuí, também observaram redução dos compostos fenólicos durante o armazenamento.

Carmo et al., (2015), Pasin et al., (2012) e Morais et al., (2015) explicam que tais resultados para fenóis podem ser decorrentes da permeabilidade da membrana que, como citado anteriormente, possivelmente proporciona a migração dos compostos por difusão osmótica, ocasionando a interação/troca entre os compostos das microcápsulas e do meio de conservação, sendo esse fenômeno denominado bioativação, do meio ou das microcápsulas. Apesar do presente estudo não avaliar os meios de conservação, a oscilação dos resultados leva a dedução dessa possível justificativa. Segundo Rezende et al., (2009) e Porto et al., (2010), outra explicação seria a análise, visto que diversos fatores podem influenciar nessa etapa, incluindo a natureza do composto, o método de extração empregado, tipo de solvente utilizado, o tempo e as condições de armazenagem, o padrão utilizado, o tamanho da amostra e a presença de interferentes tais como: ceras, gorduras, terpenos, clorofilas, vitamina C e açúcares. Quanto aos teores de betalaína nas microcápsulas, observou-se redução ao longo do armazenamento - 71,4\% para o Padrão, 66,9\% para o Ascórbico e 66\% para o Cítrico (Tabela 4). Acredita-se que tal redução se deve, possivelmente, a migração do pigmento para o meio de conservação, o que pôde ser constatado visualmente durante as análises (Figura 3). 
Figura 3: Microcápsulas de beterraba em diferentes meios de conservação. A: Ascórbico; P: Padrão; C: Cítrico

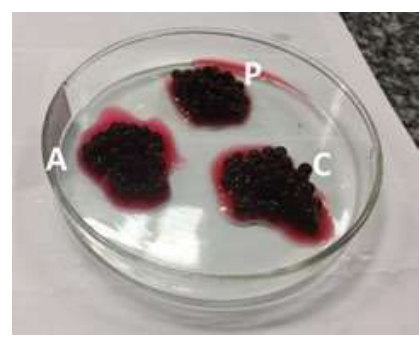

Fonte: Autores (2021).

A degradação do pigmento acarretaria na liberação do ácido betalâmico contido em sua estrutura Volp et al., (2009) e Volp et al., (2009), composto de coloração caracteristicamente amarela, ocasionando elevação dos parâmetros $b^{*}$ e H, o que não foi observado (Tabela 3). O parâmetro de cor a* evidenciou a redução do pigmento nas microcápsulas, entretanto o meio de conservação não foi analisado, sendo está uma proposta para trabalhos futuros.

O presente estudo não observou diferença significativa entre os tratamentos e o Padrão para os teores de betalaína (Tabela 4), entretanto os valores obtidos no Padrão foram inferiores aos demais tratamentos. Vitti (2003), em seu estudo de conservação de beterrabas mini processadas tratadas com ácido cítrico, observou que quanto maior o teor de ácido cítrico, menor a perda desse pigmento. O estudo de Salgado (1997), observou que a adição de ácido ascórbico (0,4\%) melhorou a estabilidade da betalaína, preservando sua concentração e coloração.

Segundo Dalla Costa (2015), a redução do teor de betalaína também acarretaria na redução dos compostos fenólicos, pois ambos os compostos apresentam forte correlação (90\%), visto que ambos são provenientes da mesma rota metabólica Almeida (2017). O presente estudo observou correlação (r) elevada entre o teor de betalaínas e fenólicos (0,80 para o Ascórbico; 0,89 para o Cítrico e 0,83 para o Padrão). Observando o comportamento dos mesmos durante o armazenamento, verificou-se elevado coeficiente de determinação $\left(\mathrm{R}^{2}\right)$ na equação polinomial (Figura 4$)$.

Figura 4: Comportamento Betalaínas X Fenólicos.

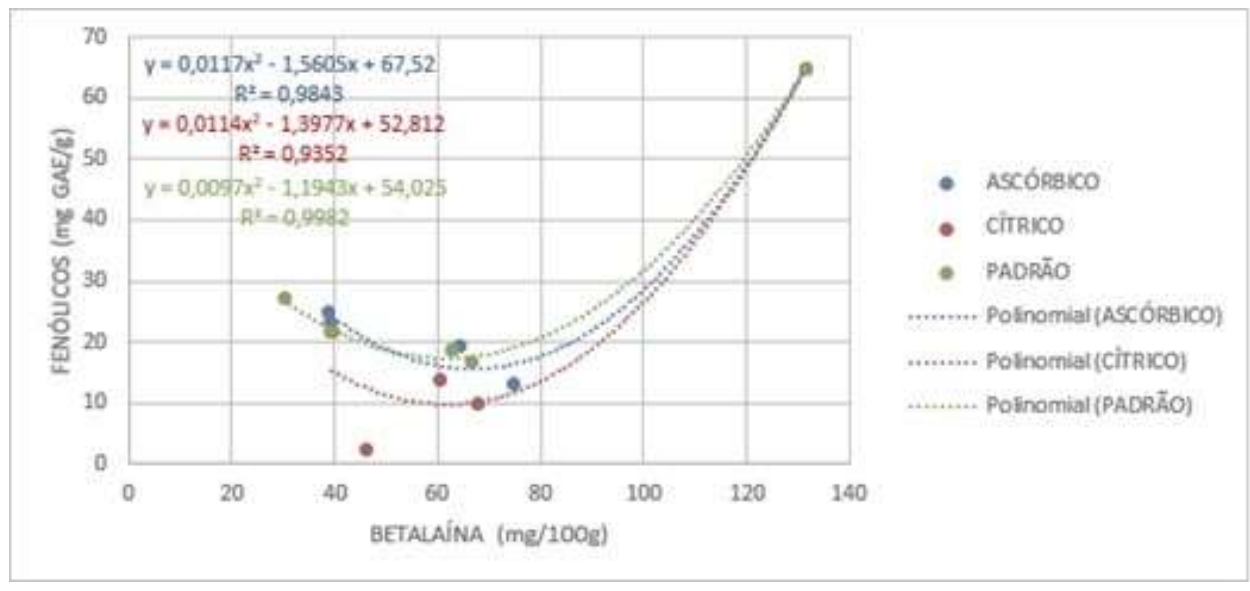

Fonte: Autores (2021).

Quanto a análise ABTS, observou-se redução da atividade antioxidante durante o armazenamento para os tratamentos e o Padrão (Tabela 5). Sendo os compostos bioativos estudados os principais responsáveis pela capacidade antioxidante da beterraba Clifford T et al., (2015) e Holkem et al., (2015), tal redução está de acordo com os resultados observados nos compostos bioativos avaliados. Os mesmos reduziram em função do tempo e aumentaram no $28^{\circ}$ dia de armazenamento, o que também pode ser observado nos resultados das análises da capacidade antioxidante pelo método ABTS. 
Tabela 5: Capacidade antioxidante $(\mu \mathrm{mol} \mathrm{TEAC} / \mathrm{g})$ pelo método ABTS das microcápsulas de extrato de beterraba armazenadas a $5 \pm 1^{\circ} \mathrm{C}$ durante 28 dias em embalagens de vidro.

\begin{tabular}{lccccc}
\hline Meio de & \multicolumn{4}{c}{ TEMPO (DIAS) } \\
\cline { 2 - 6 } armazenamento & $\mathbf{0}$ & $\mathbf{7}$ & $\mathbf{1 4}$ & $\mathbf{2 1}$ & $\mathbf{2 8}$ \\
\hline Padrão & $138,29 \pm 2,21 \mathrm{aA}$ & $125,71 \pm 2,22 \mathrm{aA}$ & $22,42 \pm 1,16 \mathrm{cC}$ & $30,02 \pm 2,35 \mathrm{cB}$ & $40,5 \pm 0,00 \mathrm{bC}$ \\
Ácido ascórbico & $138,29 \pm 2,21 \mathrm{aA}$ & $121,63 \pm 1,93 \mathrm{aA}$ & $43,14 \pm 6,01 \mathrm{cB}$ & $36,73 \pm 6,88 \mathrm{cB}$ & $73,24 \pm 6,30 \mathrm{bA}$ \\
Ácido cítrico & $138,29 \pm 2,21 \mathrm{aA}$ & $105,87 \pm 0,54 \mathrm{bA}$ & $71,19 \pm 6,93 \mathrm{cA}$ & $73,69 \pm 0,84 \mathrm{cA}$ & $58,72 \pm 7,11 \mathrm{~dB}$ \\
\hline
\end{tabular}

Médias seguidas pela mesma letra, minúscula na linha e maiúscula na coluna, não diferem entre si pelo teste de Tukey ao nível de 5\% de probabilidade.

Fonte: Autores (2021)

No $28^{\circ}$ dia de armazenamento houve aumento significativo da capacidade antioxidande das microcápsulas com Ácido Ascórbico, possivelmente pela atividade antioxidade dos compostos bioativos que permaneceram preservados nesse tratamento, como a vitamina $\mathrm{C}$, os compostos fenólicos e os carotenoides (Tabela 5).

Na Tabela 6 são descritas as correlações lineares (r) entre a atividade antioxidante pelo método ABTS e os parâmetros $\mathrm{pH}$, carotenoides, betalaínas e fenólicos.

De acordo com a Tabela 6, é possível verificar a forte correlação linear entre o teor de betalaína e a atividade antioxidante pelo método ABTS para todos os tratamentos. Tal resultado reafirma a elevada capacidade bioativa do pigmento descrita na literatura, demonstrando que quanto maior o teor de betalaínas maior o poder antioxidante Kanner et al (2001), Tesoriere et al (2004), Georgiev et al., (2010) e Gentile et al., (2004).

Tabela 6: Correlação linear entre compostos bioativos e atividade antioxidante pelo método ABTS.

\begin{tabular}{lcccc}
\hline Meio de & & \multicolumn{2}{c}{ ABTS (r) } \\
\cline { 2 - 4 } armazenamento & Carotenóides totais & Betalaína & Fenóis totais & pH \\
\hline Padrão & 0,562 & 0,775 & 0,569 & 0,913 \\
Ácido ascórbico & 0,683 & 0,797 & 0,548 & 0,845 \\
Ácido cítrico & 0,744 & 0,919 & 0,832 & 0,870 \\
\hline
\end{tabular}

Fonte: Autores (2021).

Verificou-se que apenas o tratamento com ácido cítrico apresentou forte correlação com a atividade antioxidante para carotenóides, fenólicos e betalaínas, indicando que a adição do ácido pode ter potencializado o efeito antioxidante desses compostos bioativos. Acredita-se que seu efeito acidulante tenha influenciado na redução do pH, tendo este apresentado forte correlação com o método ABTS. Tal redução, pode ter ocasionado o aumento da biodisponibilidade desses compostos e, consequente, melhora na capacidade antioxidante dos mesmos.

\section{Conclusão}

Conclui-se que é possível microencapsular o extrato de beterraba, preservando suas características nutricionais com manutenção da vitamina $\mathrm{C}$, dos compostos fenólicos, $\mathrm{pH}$, umidade, cinzas e alguns parâmetros de cor. Houve redução de carotenóides, betalaínas, parâmetro de cor $\mathrm{a}^{*}$ e atividade antioxidante. Tais resultados demonstram a viabilidade do processo de gelificação iônica, visto que as microcápsulas obtidas neste processo apresentam características nutricionais semelhantes ao extrato. Durante o armazenamento, os parâmetros dos compostos bioativos apresentaram redução. Entretanto, os dados das 
amostras conservadas em meio de ácido ascórbico apresentaram melhores resultados ao final, evidenciando que a utilização de diferentes meios de conservação pode ser uma estratégia eficaz para preservação dos compostos do extrato de beterraba microencapsulado.

As microcápsulas armazenadas em ácido cítrico apresentaram forte correlação com o método de análise antioxidante ABTS, indicando que a redução do $\mathrm{pH}$ tenha influenciado positivamente na biodisponibilidade dos compostos bioativos.

Observou-se visualmente que houve migração da cor das microcápsulas para o meio de armazenamento, sugerindo que os compostos bioativos possam também ter interagido com os meios, dessa forma sugere-se, para trabalhos futuros, a análise dos meios de conservação.

\section{Agradecimentos}

O estudo foi financiado pela Fundação de Apoio à Pesquisa e a Inovação Tecnológica do Estado de Sergipe (Fapitec/SE) - Brasil e Coordenação de Aperfeiçoamento de Pessoal de Nível Superior - Brasil (CAPES - Finance Code 001).

\section{Referências}

Almeida D. F. L. S. (2017). Estudo das Vias Metabólicas das Plantas na Síntese de Pigmentos Naturais. Dissertação de Mestrado, Universidade Fernando Pessoa.

Aranha C. P. M. (2015). Microencapsulação por gelificação iônica e interação eletrostática do corante de buriti (Mauritia flexuosa L. f.). Tese de Doutorado, Universidade Estadual Paulista "Júlio de Mesquita Filho", São José do Rio Preto, SP, Brasil.

Association of Official Analytical Chemists. (1996). Official Methods of Analysis of the Association of Official Analytical Chemists (methods 900.02). Arlington, Washington DC, Estados Unidos.

Bendich A., \& Langseth L. (1995). The health effects of vitamin C supplementation: a review. American Journal of Clinical Nutrition, 14 (2), 124-36. https://doi.org/10.1080/07315724.1995.10718484

Bosch V., Cilla A., García-Latas G., Gilabert V., Boix R., \& Alegría A. (2013). Kinetics of ascorbic acid degradation in fruit-based infant foods during storage. Journal of Food Engineering, 116 (2), 298-303. https://doi.org/10.1016/j.jfoodeng.2012.12.003.

Carmo E. L. D., Fernandes R. V. D. B., Barros R. V., \& Borges S. V. (2015). Encapsulação por spray drying, novos biopolímeros e aplicações na tecnologia de alimentos. Journal of Chemical Engineering and Chemistry, 1 (2),30-44. https://doi.org/10.18540/2446941601022015030.

Clifford T., Howatson G., West D. J., \& Stevenson E. J. (2015). The potential benefits of red beetroot supplementation in health and disease. Nutrients, 7 (4), 2801-822. https://doi.org/10.3390/nu7042801

Dalla Costa A. P. (2015). Aproveitamento de resíduos de cenoura e beterraba da indústria de minimamente processados para elaboração de ingredientes funcionais. Dissertação de Mestrado, Universidade Federal do Rio Grande do Sul, Porto Alegre, RS, Brasil.

Fang Z., \& Bhandari B. (2010). Encapsulation of polyphenols - a review. Trends in Food Science and Technology, 21 (10), 510-23. https://doi.org/10.1016/j.tifs.2010.08.003.

Fávaro-Trindade C. S., Pinho S. C., \& Rocha G. A. (2008). Revisão: encapsulação de ingredientes alimentícios. Brazilian Journal of Food Technology, 11 (2), 103-112. file:///C:/Users/Jucenir/Downloads/RevisoBJFT.pdf.

Gentile C., Tesoriere L., Allegra M., Livrea M. A., \& D'Alessio P. (2004). Antioxidant betalains from cactus pear (Opuntia ficus -indica) inhibit endothelial ICAM-1 expression. Annals of the New York Academy of Sciences, 1028 (1), 481-86. https://doi.org/10.1196/annals.1322.057.

Georgiev V. G., Weber J., Kneschke E. M., Denev P. N., \& Bley T., Pavlov A. I. (2010). Antioxidant activity and phenolic content of betalain extracts from intact plants and hairy root cultures of the red beetroot Beta vulgaris cv. Detroit dark red. Plant Foods Hum. Nutr., 65(2), 105-111. https://doi.org/10.1007/s11130-010-0156-6

Giusti M. M., \& Wrolstad R. E. (2001). Characterization and measurement of anthocyanins by UV-visible spectroscopy. Current protocols in food analytical chemistry. https://doi.org/10.1002/0471142913.faf0102s00.

Holkem A. T., Codevilla C. F., \& Menezes C. R. (2015). Emulsificação/gelificação iônica interna: Alternativa para encapsulação de compostos bioativos. Ciência e Natura, 37, 116-24. https://doi.org/10.5902/2179460X19739

Instituto Adolfo Lutz. (2008). Métodos físico-químicos para análise de alimentos. São Paulo, São Paulo, Brasil.

Kanner J., Harel S., \& Granit R. (2001). Betalains a new class of dietary cationized antioxidants. J. Agric. Food. Chem., 49 (11), 5178-185. https://doi.org/10.1021/jf010456f. 
Kim D., Jeong S., \& Lee C. Y. (2003). Antioxidant capacity of phenolic phytochemicals from various cultivars if plums. Food Chemistry, 81, 321-26. https://doi.org/10.1016/S0308-8146(02)00423-5.

Kluge R. A, \& Preczenhak A. P. (2016). Betalaínas em beterraba minimamente processada: Perdas e formas de preservação. Revista Iberoamericana de Tecnología Postcosecha, 17 (2). https://www.researchgate.net/publication/314172659.

Krasaekoopt W., Bhandari B., \& Deeth H. (2003). Evaluation of encapsulation techniques of probiotics for yogurt. Internacional Dairy Journal, 13 (1), 3-13. https://doi.org/10.1016/S0958-6946(02)00155-3.

Lacerda Y. E. R. (2014). Produção e qualidade de cenouras e de beterrabas com aplicação de fertilizantes orgânicos. Dissertação de Mestrado, Universidade Estadual da Paraiba, Campina Grande, Paraiba, Brasil.

Lichtenthaler H. K. (1987). Chlorophylls and carotenoids: pigments of photosynthetic biomembranes. Methods in enzymology, 148, 350-82. https://doi.org/10.1016/0076-6879(87)48036-1.

Marques L. F., Medeiros D. C. D., Coutinho O. D. L., Marques L. F., Medeiros C. D. B., \& Vale L. S. D. (2010). Produção e qualidade da beterraba em função da adubação com esterco bovino. Revista Brasileira de Agroecologia, 5 (1), 24-31. http://revistas.abaagroecologia.org.br/index.php/rbagroecologia/article/view/7602.

Morais A. B. L, Xavier A. C. R., Silva G. F., Muniz E. M., Muniz A. V. C. S., \& Pagani A. A. C. (2014, setembro). Efeito da encapsulação nos compostos bioativos do cambuí (Myrciaria tenella), Anais do XXIV Congresso Brasileiro de Ciência e Tecnologia de Alimentos, Aracaju, Sergipe, Brasil, 14.

Mirzaei H., Pourjafar H., \& Homayouni A. (2012). Effect of calcium alginate and resistant starch microencapsulation on the survival rate of Lactobacillus acidophilus La5 and sensory properties in Iranian white brined cheese. Food Chemistry, 132 (4), 1966-970. https://doi.org/10.1016/j.foodchem.2011.12.033.

Morais A. B. L., Xavier A. C. R., Silva G. F., Silva M. A. A. P., \& Pagani A. A. C. (2015). Bioactivation of carbonated mineral water with passion fruit microcapsules. International Journal of Nutrition and Food Sciences, 4 (3), 310-19. https://doi.org/10.11648/j.ijnfs.20150403.18

Morais A. B. L., Xavier A. C. R., Silva M. A. A. P., Souza R. R., Pagani G. D., \& Pagani A. A. C. (2017, janeiro). Caracterização da polpa e das cápsulas de murici (Byrsonima crassifolia L. Rich) obtidas pelo processo de gelificação iônica. Anais do XIII Congresso Internacional de Nutrição Funcional, São Paulo, São Paulo, Brasil, 13.

Neto J. F., Queirós M. M. F., Nobre R. G., Pereira Junior E. B., Sousa J. C., \& Sousa J. X. (2017). Caracterização físico-química e microbiológica da beterraba irrigada com efluente agroindustrial. Rev. de Agroec. no Semiárido, 1 (1), 13-23. https://periodicos.ifpb.edu.br/index.php/ras/article/viewFile/41-2PB\%20pdf/artigo.

Oliveira M. C. (2011). Estudo do processo de obtenção de gotas de mamão (carica papaya L.) por esferificação. Dissertação de Mestrado, Universidade Federal de Sergipe, São Cristóvão, Sergipe, Brasil.

Oliveira R. G. D., Godoy H. T., \& Prado M. A. (2010). Otimização de metodologia colorimétrica para a determinação de ácido ascórbico em geleias de frutas. Food Science and Technology, 30(1), 244-249. https://www.scielo.br/j/cta/a/RnCX3hMMxLcPzwmVJ7csGVz/?lang=pt\&format=pdf.

Pagani A. A. C., Oliveira M. C., Xavier A. C. R., Morais A. L. B., Nunes T. P., \& Silva G. F. (2014). Study of the process of getting the drops of papaya (carica papaya) per basic spherification. International Journal of Engineering and Innovative Technology (IJEIT), 4 (6), 1-9. https://www.ijeit.com/Vol\%204/Issue\%206/IJEIT1412201412_01.pdf.

Pasin B. L., Azón C. G., \& Garriga A. M. (2012). Microencapsulación con alginato en alimentos. Técnicas y aplicaciones. Revista Venezolana de Ciencia y Tecnología de Alimentos, 3 (1), 130-51. http://oaji.net/articles/2017/4924-1495374245.pdf.

Passos R.M. (2017). Incorporação da polpa de tomate encapsulada por gelificação iônica ao azeite de oliva extravirgem e avaliação da estabilidade do produto. Dissertação de Mestrado, Universidade Federal de Sergipe, São Cristóvão, Sergipe, Brasil.

Palou E., López-Malo A., Barbosa-Cánovas G. V., Welti-Chanes J., \& Swanson B.G. (1999). Polyphenoloxidase activity and color of blanched and high hydrostatic pressure treated banana puree. Journal of Food Science, 64, 42-45. https://doi.org/10.1111/j.1365-2621.1999.tb09857.x.

Porto R. G. C. L., Cunha E. M. F., Barros N. V. A, Silva M. G. S. S, \& Moreira-Araújo R. S. R. (2010). Correlação entre a capacidade antioxidante e o conteúdo de vitamina $\mathrm{C}$, antocianinas, flavonóides e fenólicos totais no Jenipapo (Genipa americana L.). Universidade Federal do Piauí. 1-4. http://leg.ufpi.br/20sic/Documentos/RESUMOS/Modalidade/Vida/390e982518a50e280d8e2b535462ec1f.pdf?fbclid=IwAR0b3YwZU_k0t2QZp8MRAAsk_f ZGMp6_1GWy_YjUzuZOp2xXG20ijyBgDzA.

Raupp D. D. S., Rodrigues E., Rockenbach I. I., Carbonar A., Campos P. F. D., Borsato A. V., \& Fett R. (2011). Effect of processing on antioxidant potential and total phenolics content in beet (Beta vulgaris L.). Food Science and Technology, 31 (3), 688-93. https://doi.org/10.1590/S0101-20612011000300021

Rezende L. C., Oliveira T. S., Alves C. Q., David J. M., \& David J. P. (2009, julho). Fenólicos totais e atividade antioxidante de frutas tropicais da Bahia, Anais da $32^{\circ}$ Reunião anual da Sociedade Brasileira de Química, Bahia, Salvador, Brasil, 32.

Rufino M. S. M., Alves R. E., de Brito E., de Morais S. M., de Sampaio C. G., Pérez-Jiménez J., \& Saura-Calixto F. D. (2007). Metodologia Científica: determinação da atividade antioxidante total em frutas pela captura do radical livre. Comunicado Técnico online n ${ }^{128}$. Fortaleza, Ceará, Brasil.

Salgado S. M. A. (1997). Estudo da estabilidade de betalaína extraída da beterraba-vermelha de mesa (Beta vulgaris L.). Dissertação de Mestrado, Universidade Federal de Viçosa, Viçosa, MG, Brasil.

Schiozer A. L., \& Barata L. E. S. (2013). Estabilidade de corantes e pigmentos de origem vegetal. Revista Fitos Eletrônica, 3 (2), 6-24. https://revistafitos.far.fiocruz.br/index.php/revista-fitos/article/view/71.

Sezer A. D, \& Akbuga J. (1999). Release characteristics of chitosan treated alginate beads: I. Sustained release of a macromolecular drug from chitosan treated alginate beads. Journal of Microencapsulation, 16 (2), 195-203. https://doi.org/10.1080/026520499289176 
Research, Society and Development, v. 10, n. 12, e454101220171, 2021

(CC BY 4.0) | ISSN 2525-3409 | DOI: http://dx.doi.org/10.33448/rsd-v10i12.20171

Silva E. A., Lima J. S., Santos E. A. L., Araujo J. M., Machado C. T., \& Castro A. A. (2012). Desenvolvimento e avaliação das características físico-químicas de produtos encapsulados à base de melancia (Citrullus lanatus). Anais do III Simpósio Internacional de Plantas Medicinais e Nutracêuticas (3ISMNP) e III Conferência do Instituto Nacional de Ciência e Tecnologia de frutos Tropicais. Aracaju, Sergipe, Brasil, 3.

Silva W. L., Medeiros R. A. B. D., Pires E., \& Freitas M. (2016). Eficiência do cloro para sanitização de hortaliças. Hig. Alimente., 30 (256/257), 132-36. http://www.ufrgs.br/sbctars-eventos/gerenciador/painel/trabalhosversaofinal/SAL439.pdf.

Singh B., Singh J. P., Kaur A., \& Singh N. (2017). Phenolic composition and antioxidant potential of grain legume seeds: A review. Food Research International, 101,1-16. https://doi.org/10.1016/j.foodres.2017.09.026.

Sucupira N. R., Xerez A. C. P., \& de Sousa P. H. M. (2015). Perdas vitamínicas durante o tratamento térmico de alimentos. Journal of Health Sciences, 14 (2), 121-128. https://doi.org/10.17921/2447-8938.2012v14n2p\%25p

Tesoriere L., Allegra M., Butera D., \& Livrea M. A. (2004). Absorption, excretion, and distribution of dietary antioxidant betalains in LDLs: Potential health effects of betalains in humans. Am. J. Clin. Nutr., 80 (4), 941-45. https://doi.org/10.1093/ajcn/80.4.941.

Tivelli S. W., Factor T. L., Teramoto J. R. S., Fabri E. G., Moraes A. R. A., Trani P. E., \& May A. (2011). Beterraba: do plantio à comercialização. (Série Tecnologia APTA. Boletim Técnico IAC, 210), Campinas, SP, Instituto Agronômico.

Vitti M. C. D. (2003). Aspectos fisiológicos, bioquímicos e microbiológicos em beterrabas minimamente processadas. Tese de Doutorado, Universidade de São Paulo, São Paulo, São Paulo, Brasil.

Volp A. C. P., Renhe I. R. T., \& Stringueta P. C. (2009). Pigmentos naturais bioativos. Alimentos e Nutrição Araraquara, 20 (1), 157-66. http://servbib.fcfar.unesp.br/seer/index.php/alimentos/article/view/959/786.

Xavier A. C. R. (2014). Pérolas de maracujá obtido por processo de gelificação iônica. Dissertação de Mestrado, Universidade Federal de Sergipe, São Cristóvão, Sergipe, Brasil.

Xavier A. C. R., Morais A. B. L., Silva D. P., Silva G. F., \& Pagani A. A. C. (2016, setembro). Pérolas de polpa de maracujá obtidas por gelificação iônica, Anais do VII International Symposium on Technological Innovation: Innovation to Inspire and Implement. Aracaju, Sergipe, Brasil, 7. 